\title{
Cartografía social del discurso académico sobre la extensión en la Universidad Nacional de Costa Rica: perspectivas exploratorias
}

\section{Social Cartography of Academic Discourse on University Extension at the National University of Costa Rica: Exploratory Perspectives}

\author{
Francisco González Alvarado \\ Universidad Nacional \\ Heredia, Costa Rica \\ francisco.gonzalez.alvarado@una.cr
}

Recibido: 28/05/19 Aceptado: 30/08/19

Resumen. El ejercicio de la extensión social universitaria como labor sustantiva constituye un escenario discursivo y práctico siempre complejo, dinámico y ante todo consecuencia del diálogo con la sociedad. El texto presenta los resultados de un trabajo indagatorio sobre el discurso académico de la extensión en la Universidad Nacional, a partir del empleo de los principios metodológicos de la cartografía social. Analiza para ello, principalmente, un conjunto seleccionado de textos producidos por un grupo de académicos distinguidos por la institución con la categoría de «extensionistas destacados», identificando diferentes perspectivas exploratorias discursivas al interior de su producción intelectual, generada a través de diferentes décadas. El propósito central del escrito es construir un mapa exploratorio del discurso sobre la extensión universitaria que permita identificar los rasgos distintivos de la naturaleza y carácter propio de esta forma de la acción sustantiva en la Universidad Nacional de Costa Rica.

Palabras clave: dimensiones discursivas, cartografía social, extensión universitaria.

Abstract. The exercise of university social extension as a substantive work constitutes a discursive and practical scenario that is always complex, dynamic, and, above all, a consequence of a dialogue with society. The text shows the results of research work on the academic discourse of the university extension at the National University, based on the use of the methodological principles of social cartography. For this, the paper 
mainly analyzes a selected set of texts, produced by a group of scholars distinguished by the institution with the category of "outstanding extension workers." It identies different exploratory discursive perspectives within their intellectual production, generated over various decades. The primary purpose of the writing is to build an exploratory map of the discourse on university extension which, allows identifying the distinctive features of the nature and character of this form of substantive action at the National University of Costa Rica.

Keywords: discursive dimensions, social cartography, university extension.

\section{Introducción}

Todo intento por examinar con visión histórico-prospectiva un ámbito de la acción sustantiva universitaria: docencia, investigación o extensión, pasa por la necesaria revisión de las diferentes perspectivas discursivas reflejadas en la producción intelectual de su comunidad académica y cuyo acopio documental es posible ubicarlo de manera más o menos sistemática, en las diferentes publicaciones institucionales generadas a través de los años. Es por medio de su identificación y posterior análisis que es posible aproximarse a reconocer principios de naturaleza teórico-prácticos, metodológicos, filosóficos, epistémicos y políticos, así como enfoques y concepciones sobre una u otra forma del quehacer académico y su vinculación con el modelo de universidad asumido en determinado contexto histórico. Para el caso de la extensión universitaria, la tarea descrita es un tanto más compleja, especialmente porque la sistematización documentada de las experiencias no es tan común de hallar, a diferencia, por ejemplo, de las relacionadas con la investigación o la docencia misma, donde los registros son más abundantes.

Al respecto, este texto expone los resultados de un trabajo indagatorio, realizado durante el 2018, sobre el análisis del discurso académico de la extensión en la Universidad Nacional de Costa Rica (UNA), empleando principios metodológicos de la cartografía social y cuyo propósito se centró en identificar de manera exploratoria la naturaleza y carácter propio de esta forma de la acción sustantiva universitaria. Para ello, analiza la producción intelectual de un grupo de académicas y académicos que se han distinguido por su trabajo denodado y de largo tiempo en la extensión universitaria. Los resultados permitieron identificar, por medio de la metodología citada, cuatro perspectivas exploratorias principales del discurso sobre la extensión en la UNA. Todas, configuraciones conceptuales que posteriormente se localizaron en el marco de la construcción de un mapa-dibujo que expresa el espacio o territorio discursivo. 
En este sentido, «...lo que vamos a mencionar como "mapa" no es de facto el mapa tradicional sino un compuesto complejo elaborado a partir del diálogo, la memoria, las representaciones subjetivas, el conocimiento y las singularidades constituyentes en "un plano común” ... « (Tetamanti, y Chanampa, 2016, p. 86).

La localización en el mapa-dibujo de las perspectivas exploratorias señaladas y su posterior caracterización, tal como se expondrá en detalle, evidenció posiciones discursivas alrededor de la extensión, más complementarias que discrepantes, sin que lo anterior estreche la significativa riqueza conceptual y epistémica hallada alrededor del discurso académico de la extensión en la UNA.

\section{Referentes teóricos}

\section{Discusiones sobre los modelos de la extensión universitaria}

La universidad pública latinoamericana, fundamentalmente a partir de la Reforma Estudiantil de Córdoba de 1918, ha destacado por su acento en el desarrollo de la extensión social como parte esencial de su acción sustantiva. Precepto que evidencia el sentido político, teleológico y el marco ético de su quehacer. Tal preocupación se expresa con suficiente claridad en prácticamente todas las declaratorias estatutarias de las universidades públicas de la región, que contemplan entre sus principios, fines y valores la contribución decidida en la transformación de las sociedades desde una perspectiva democrática e inclusiva a través de un mayor acercamiento (comunicación) con la identificación y solución a los problemas históricos y coyunturales de las poblaciones más excluidas.

Sin embargo, no puede sostenerse el argumento de que esa forma de participación social constituya un corpus homogéneo, por el contrario, tanto desde el discurso como en la práctica, exhibe diferentes posiciones o modelos. De ahí que se considere la discusión sobre la extensión universitaria como un terreno teórico en disputa.

$\mathrm{Al}$ respecto, Serna (2007) reconoce cuatro modelos de extensión universitaria. El altruista: que emerge junto a la concepción de extensión emanada alrededor de los postulados de la Reforma de Córdoba e impulsa la acción desinteresada y humanitaria de las universidades a favor de los sectores más desprotegidos. Su orientación proclama la obligación de llevar los conocimientos de la universidad al pueblo, con el sentido de corresponder a los esfuerzos de la sociedad en materia de inversión en educación superior. El divulgativo: según el autor mencionado, es la noción de «extensión» que mayor peso ha tenido. Parte de la concepción de que la ciencia y la cultura universitaria deben «rebajarse» para ser comprendidas por el pueblo, utilizando atractivamente los medios de 
comunicación. El concientizador: diferente a los anteriores, este modelo propone que la extensión universitaria sea una herramienta educativa transformadora de la sociedad. Recoge las ideas de la izquierda latinoamericana y se enriquece puntualmente con el pensamiento de Pablo Freire, para quien el papel central de la educación es el de compartir los bienes, incluidos los económicos y culturales, de forma dialógica, crítica, transformadora y liberadora. El último modelo es el vinculatorio empresarial: en este las necesidades sociales son identificadas como análogas a las del mercado y de las empresas privadas, en consecuencia, la extensión universitaria se orienta a cubrir tales demandas.

Otra perspectiva sobre los modelos de la extensión universitaria es la aportada por González y González (2003 y 2006), citados por Ortiz y Morales (2011), a partir de lo que denominan las prácticas de las universidades latinoamericanas. A diferencia de la clasificación de Serna (2007) mencionada en el párrafo anterior, esta incluye tres: el modelo tradicional: mediante el cual la universidad se concibe como fuente de conocimiento y saberes, y establece una relación de saber institucionalizado con quien no lo posee. Este modelo tiene similitudes con el primero que plantea Serna, en el que el altruismo es el principio que guía las acciones. El segundo es el modelo economicista: en este, la universidad se concibe como una empresa más que interactúa en el mercado. Adquiere el rol de soporte científico y técnico del sector productivo, y el saber se organiza en función de la rentabilidad económica. Postura similar a la expuesta en el modelo vinculatorio empresarial. El último es el modelo de desarrollo integral: la universidad maneja el concepto de democratización del saber y asume la función social de contribuir a mejorar la calidad de vida de la sociedad. Apunta a la transformación social y económica de los pueblos. Las dos clasificaciones sobre los modelos de la extensión descritas, más que antagónicas, son complementarias y constituyen un ejercicio reflexivo importante de tomar en cuenta, especialmente porque permite ordenar y analizar el discurso sobre la extensión universitaria desde diferentes marcos referenciales, cuestión central para la realización del análisis acometido y la caracterización de las diferentes perspectivas halladas.

\section{Metodología}

Con el propósito de identificar, caracterizar y discutir las dimensiones centrales del discurso académico sobre la extensión en la UNA, se empleó la metodología de la cartografía social, entendida como «un enfoque metodológico basado en el análisis textual que busca la representación de fenómenos sociales a través de mapas que reinscriben y estructuran una multiplicidad de perspectivas o 
URL: http://www.revistas.una.ac.cr/index.php/dialogo/index

CORREO ELECTRÓNICO: universidadendialogo@una.cr

DOI: http://doi.org/10.15359/udre.9-2.4

formas de ver estos fenómenos» (Tello y Gorostiaga, 2009, p. 159). También, definida por Tetamanti y Chanampa (2016) como:

...una máquina deleuziana de producción generadora de mapas, representaciones colectivas y transformaciones a partir de la producción de nuevos sentidos espaciales (Debord y Wolman, 1956). Es decir, un método de proceso maquínico en el cual intervienen sí o sí más de dos sujetos, los cuales producen, a partir de la intertextualidad de experiencias y prácticas, sobre el mundo a representar y, al mismo tiempo: generan nuevos sentidos espaciales que incrementan el conocimiento de los partícipes sobre ese espacio y, en oportunidades, colaboran en el obrar sobre él. (p. 86)

Esta orientación sistemática de investigación siguiendo a los autores mencionados favorece identificar e interpretar de manera organizada e intencionada diferentes puntos de vista alrededor de una determinada área de conocimiento o campo discursivo, para posteriormente lograr una representación provisional y exploratoria de las principales dimensiones argumentativas y cosmovisiones que prevalecen entre un determinado grupo de representantes o exponentes, incluyendo sus similitudes y diferencias más significativas. Por esto la pertinencia del enfoque para el análisis del discurso académico de la extensión en la UNA.

Es oportuno señalar también que la cartografía social es útil para el análisis de textos y sus relaciones con otros (intertextualidad) en un determinado contexto histórico-discursivo como principal herramienta, y cuyo propósito central es el de identificar las diferentes perspectivas presentes y proceder a su análisis integrador. Lo que supone un proceso interactivo que incluye «lectura de los textos; identificación provisional de las principales perspectivas; selección de los textos ilustrativos de cada perspectiva; lectura en profundidad de los textos seleccionados; revisión de las perspectivas identificadas; incorporación/ eliminación de textos ilustrativos» (Tello y Gorostiaga, 2011, p. 364).

A partir de los presupuestos metodológicos descritos se identificaron, seleccionaron y analizaron veintiocho textos en total. De ellos, dieciocho corresponden a un grupo de académicos con el reconocimiento institucional de «extensionistas destacados» (ver anexo 1). Mérito otorgado luego de cumplir para su elección "con criterios de excelencia y pertinencia de sus acciones, resultados conocidos en sus programas, proyectos y actividades de extensión, constancia y creatividad en el desarrollo de sus iniciativas» (Universidad Nacional, Vicerrectoría de Extensión, 2016, p. 1). Condiciones que justifican la representatividad del grupo en el quehacer académico de la 
extensión en la UNA, específicamente porque el aporte discursivo registrado ha sido prolongado en el tiempo, abundante y representativo.

Los diez restantes (ver anexo 2) constituyen publicaciones que, por el nivel de especificidad, profundidad y referencia institucional con el que abordan directamente y en forma singular el tema de análisis, se consideraron pertinentes de incluir. Los documentos constituyen textos publicados mayoritariamente en medios escritos de la UNA, entre 1980 y el 2018, los cuales se ubicaron mediante diferentes fuentes bibliográficas institucionales: el Portal Electrónico de Revistas Académicas, el Repositorio Académico, el Sistema de Información Documental de la Universidad Nacional (SIDUNA) y Google Académico. La producción intelectual incluye artículos de revistas, ponencias, libros, monografías e informes de proyectos.

El análisis de todos los textos, en consecuencia con la metodología señalada, incluyó la identificación de los argumentos medulares mediante la guía exploratoria de cuatro preguntas sobre la extensión universitaria: ¿Qué es?, ¿qué propósitos pretende?, ¿qué sentido le otorga dentro del quehacer de la universidad? y ¿cuáles son los principios metodológicos principales? Los registros de las respuestas se levantaron mediante matrices clasificatorias, en las cuales se integró el nombre de los autores, la ficha bibliográfica correspondiente y las transcripciones textuales. Identificados los argumentos, se procedió a su yuxtaposición mediante la construcción de las cuatro perspectivas académicas discursivas (núcleos semánticos) consideradas centrales sobre la extensión universitaria.

Posteriormente, se procedió a la construcción de dos mapas-dibujos (territorios discursivos) con apoyo de la herramienta CmapsTool 6.2, ambos sobre la selección de dos dimensiones (categorías que delimitan el territorio), que incluyen dos polos epistémico-conceptuales cada uno. Partiendo de que la «...representación visual del campo intertextual se realiza a través de un mapa construido en base a las dos dimensiones que surgen como las más significativas en el posicionamiento y diferenciación de las perspectivas identificadas» (Tello y Gorostiaga, 2011, p. 365).

\section{Resultados}

\section{Perspectivas del discurso académico sobre la extensión en la UNA}

El mapa-dibujo provisorio de la cartografía social del discurso académico sobre la extensión en la UNA (ver figura 1) se ordenó mediante la delimitación de dos dimensiones, la vertical expresa el sentido misional de la extensión, es decir, su propósito medular dentro del quehacer sustantivo de la universidad 
URL: http://www.revistas.una.ac.cr/index.php/dialogo/index

CORREO ELECTRÓNICO: universidadendialogo@una.cr

DOI: http://doi.org/10.15359/udre.9-2.4

y la horizontal permite identificar las formas-estrategias de vinculación de esta con la sociedad. Cada una de ellas se articula con dos respectivos polos epistémico-conceptuales vinculados con modelos teóricos explicativos de la extensión latinoamericana.

La vertical (sentido misional) incluye en el polo norte el modelo concientizador (Ortiz y Morales, 2011) y en el extremo sur el tradicional (González y González, 2003). Y la horizontal (formas-estrategias de vinculación social) en el polo este incluye el modelo de desarrollo integral y en el opuesto el economicista, siguiendo la propuesta de González y González (2003). Al interior del territorio configurado se identifican cuatro perspectivas centrales del discurso académico sobre la extensión: la transformación social, la comunicación cultural, la acción sustantiva y la vinculación social orgánica. Estas se representan con óvalos de colores de contorno punteado, y en rectángulos del mismo color los respectivos núcleos conceptuales explicativos de cada una.

La posición de las perspectivas dentro de las dimensiones del territorio y los correspondientes polos gravitan en función de su aproximación semántica, manifestando una particular atracción hacia el modelo concientizador y el de desarrollo integral, a pesar de que algunos de los núcleos conceptuales, especialmente los asociados con la vinculación social orgánica, expresan cierta cercanía con los modelos tradicional y economicista.

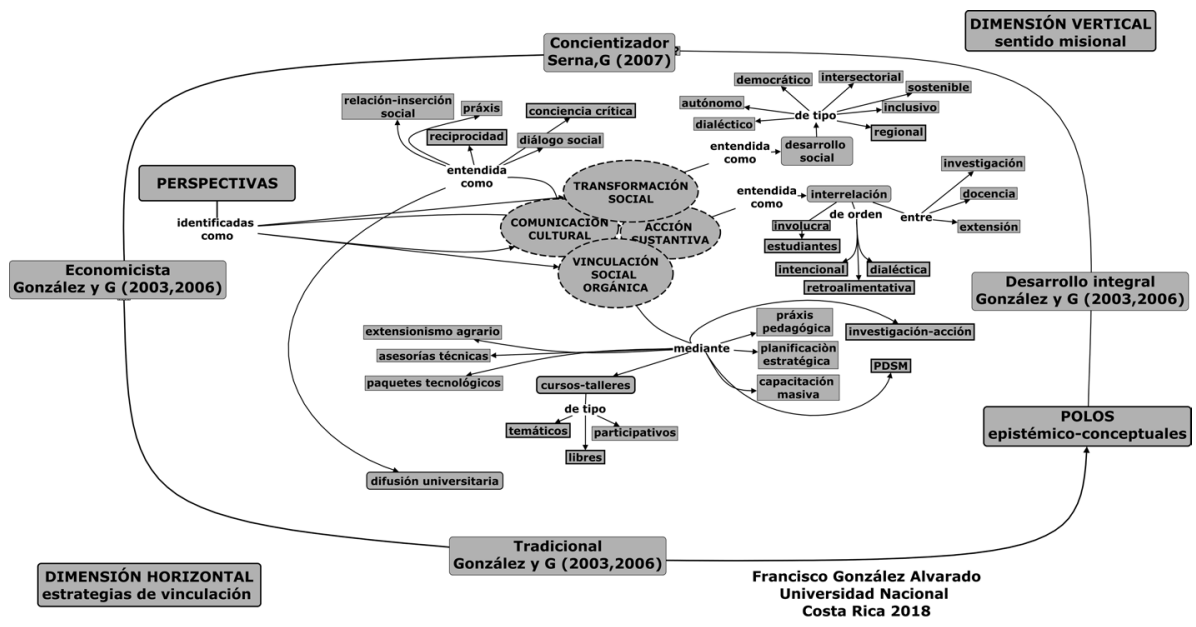

Figura 1. Cartografía de las perspectivas sobre la extensión en la UNA.

Fuente: Francisco González Alvarado (2018). 


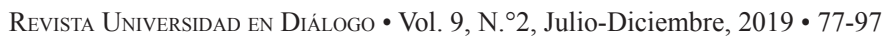

ISSN 2215-2849 • EISSN: 2215-4752

URL: http://www.revistas.una.ac.cr/index.php/dialogo/index CoRReo eleCtrónico: universidadendialogo@una.cr DOI: http://doi.org/10.15359/udre.9-2.4

Seguidamente, se presenta una breve caracterización de cada una de las perspectivas halladas, con el propósito de identificar rasgos centrales de la naturaleza y carácter propio del discurso académico de la extensión. También se hace uso de citas textuales como recurso ilustrativo de aquellos elementos considerados relevantes.

\section{Transformación social}

Esta cosmovisión del discurso de la extensión en la UNA se encuentra estrechamente reflejada en los principios misionales originarios de la universidad y es compartido con suficiente énfasis en los textos de Fallas, López y Meoño (1982), Goldemberg (1988), Sobrado (2011), Aguirre (2017), Bonilla (2010), Brenes (2006), Gamboa (2013), Núñez (1974) y Ramírez (1998). La precisa vinculación entre la transformación social, el desarrollo social y el papel promotor de la universidad en el logro de este acometido constituye un elemento central de esta perspectiva.

Al respecto, en el texto considerado fundacional de la UNA, el padre Benjamín Núñez, su primer rector, expresa: «Se afirma que la universidad democratiza el modelo de desarrollo en cuanto contribuye a orientarlo hacia el bienestar de todos, con base en una amplia participación popular y sin detrimento de los derechos de cualquier sector de la comunidad nacional» (Núñez, 1974) p. 26). Este argumento es propio de la filosofía liberadora latinoamericana surgida en la década de los setenta, cuya preocupación era «...crear un instrumental ideológico que logre romper los resortes de la discriminación impuesta de las formas de dependencia» (Santos, 2010, p. 167).

La perspectiva de la transformación social expresa un significado triple, por un lado, se plantea como una estrategia posible para reconocer los problemas sustantivos de la sociedad, por otro, considera el impulso de las transformaciones necesarias que permitan atenderlos, al facilitar acciones particulares de superación de las condiciones de exclusión social históricamente construida, con especial énfasis en el ámbito del desarrollo rural. Al respecto, Aguirre (2016) expone con suficiente claridad, sustentado en una experiencia concreta:

El Plan Desarrollo de la Comunidad de Barra de Colorado consta de un diagnóstico participativo sobre la situación de los sectores estratégicos de la comunidad. Luego se efectuó un análisis integral de la situación de la comunidad, posteriormente se realiza una proyección de la situación de la comunidad, para ello se construyen dos escenarios en el tiempo: pesimista y optimista. Se proyecta la visión de desarrollo que se desea alcanzar (se integran los principios, misión, objetivos y políticas). (p. 209) 
Finalmente, también contempla el ineludible compromiso transformador sobre la universidad misma, desde un posicionamiento de carácter dialéctico. Fallas, López y Meoño (1982) profundizan y amplían esta última postura:

En realidad, la transformación social que la Universidad puede apoyar, actúa al mismo tiempo, como factor de su propia transformación y desarrollo. A medida que se favorece la transformación social, se enriquece la transformación interna de la Universidad. De la misma manera, al basar su propio desarrollo en la transformación social, la vinculación consciente de la Universidad, y la sociedad permite una incidencia y una retroalimentación mucho más efectivas. (p. 142)

Esta perspectiva de la extensión universitaria también distingue una serie de principios condicionantes para propender a la transformación social, factores subyacentes que delimitan el ejercicio de la labor sustantiva por parte de los involucrados, estos son: el carácter inclusivo, el democrático, el equitativo, el endógeno, el sostenible, el planificado, el autónomo, el intersectorial y el de amplia participación social. Todos entendidos como preceptos ineludibles y componentes esenciales de la relación dialéctica universidad-sociedad. Ramírez (1998) aporta argumentos a favor de esta postura:

A partir de este accionar conjunto, UNA-Comunidad, se integraron otros grupos de apoyo, con el fin de unir esfuerzos para llevar a cabo una serie de programas tendientes a alcanzar un verdadero desarrollo comunal (...) el trabajo comunitario se realizó con niños de edad escolar, jóvenes, docentes, líderes comunales y amas de casa. (p. 14)

De igual forma, Ruiz (2011) sostiene que:

La participación de la población sujeto es fundamental en este proceso de ejecución y búsqueda de soluciones a los problemas que se van presentando y permanente negociación con las diferentes instituciones que se comprometieron a trabajar. Este proceso enriquecedor los facultará para los futuros proyectos que tendrán que formular, negociar y desarrollar sin la participación de la Universidad. (p. 27)

\section{Comunicación cultural}

Otra perspectiva plantea que la extensión es una forma de comunicación cultural. Esta postura es desarrollada con mucha profundidad en los textos de Núñez (1974), Ruiz (1992) y Rivera (1983), quienes la asocian con el concepto de 
difusión cultural, en su acepción más crítica, sobre todo los dos primeros. Acá se le asigna a la cultura en su acepción más amplia un papel central en la comprensión y transformación de los fenómenos y las realidades sociales, económicas y políticas. Aparece vinculada directamente con la noción de unidad de cultura, entendida como «el conjunto de valores y prácticas que constituyen al ideal ético-racional en el cual se fundamente el esfuerzo de propósitos de una nación» (Núñez, 1974 p. 12). Tal postura ideológica, filosófica, ética y política aboga por la necesidad de que los «propósitos de una nación» se amalgamen hacia la consecución de planos de mayor bienestar y participación democrática de sus integrantes.

Esta visión se presenta como la forma mediante la cual el quehacer de la universidad también es concebido como expresión cultural y es vinculado, comunicado, más que extendido, a la sociedad y expresa su compromiso con su construcción-transformación. En el contexto discursivo señalado la comunicación cultural se aparta de la visión de transmisión, considerada carente de sentido ético-político del quehacer universitario con la sociedad y en contraparte aboga por otro centrado en los procesos de comunicación universidad-sociedad, distinguiendo su carácter continuo, permanente, dinámico y cambiante, tal como lo señala Ruiz (1992).

La misma autora plantea, para diferenciar y asegurar su consecución, una jerarquización por niveles de menor a mayor grado, distinguiendo cinco: información, relaciones públicas, difusión del quehacer académico, difusión cultural universitaria y finalmente extensión. Los objetivos que distinguen cada uno de los niveles permiten valorar la orientación de las prácticas de la extensión, exponiendo las diferencias entre las más simples asociadas a procesos típicamente de información y acercamiento de su quehacer con la sociedad, a otras donde se impulsa la visión crítica, concientizadora, de transformación social y se defiende una nueva unidad de cultura.

El sentido de la extensión universitaria entendida como comunicación cultural crítica y concientizadora distingue una serie de principios de carácter epistémico y metodológico, donde resaltan la inserción intencionada de la universidad en la sociedad y el carácter praxeológico de esa forma de vinculación. El primero reconoce la preponderancia de atender a los grupos sociales más vulnerables:

La labor académica incluye la responsabilidad ética que transciende las aulas de los campus universitarios. Esta participación busca el desarrollo local orientado al mejoramiento de la calidad de vida de los grupos sociales que habitan un determinado espacio territorial y el fortalecimiento de las capacidades humanas con visión de futuro. (Gamboa, 2013, p. 322) 
El segundo enfatiza en el aporte sustantivo de esa comunicación respecto al impacto social y la relación entre los universitarios y los grupos sociales, al respecto Ramírez (2011) expresa:

...el proyecto fortaleció la capacidad de organización, gestión y liderazgo de los grupos involucrados. Asimismo, planteó el mejoramiento de las destrezas y habilidades de apicultores y apicultoras mediante un proceso de capacitación que comprendió las principales fases de la cadena agroproductiva. (p. 43).

Un logro importante, fue incentivar la participación activa de las mujeres en los procesos productivos de la empresa apícola y fomentar el desarrollo sostenible de zonas rurales marginales de nuestro país, mediante la generación de nuevas fuentes de empleo y el incremento de los ingresos favorable y contribuyó a mejorar la conservación ambiental y las condiciones socioeconómicas de la Región Central Sur de nuestro país. (p. 52)

Tales reflexiones conceptuales se asocian a la perspectiva elaborada por Paulo Freire en todo su trabajo intelectual sobre la estrecha asociación y diferenciación a la vez entre la extensión, explicada como acto mecánico y autoritario, y la comunicación, expresada como diálogo democrático, problematizador y liberador.

\section{Acción sustantiva}

Esta tercera perspectiva pone el énfasis en la importancia de reconocer la extensión como una forma de la acción sustantiva de la universidad, discute su vinculación con la docencia y la investigación y plantea aspectos de su relación con el nivel organizativo institucional. Los principales referentes discursivos sobre este posicionamiento se registran en los trabajos de Hernández (1980), Rivera (1983), Fallas, López y Meoño (1982), Ruiz (1992) y León (2007). Los textos argumentan con profundidad a favor del carácter esencialmente académico de la extensión universitaria. Hernández (1980), al respecto, argumenta con meridiana claridad:

La Extensión Universitaria es, debe ser, una irrenunciable y prioritaria exigencia socioacadémica que busca y se propone que los grupos o sectores sociales más postergados e indigentes actúen, confiando y realizándose, como sujetos de su propia libertad y capacidad. Extensión no puede ser transmisión mecánica desde la «sede del saber» hasta la «sede de la ignorancia», debe ser diálogo que lleve a lograr conciencia crítica de la realidad e interacción educadora dentro de la realidad 
que los sectores, las comunidades y los grupos particulares quieren transformar consciente y responsablemente. (p. 66)

De igual forma, discuten las condiciones académicas para lograr esa materialización; importancia de la sistematización de las experiencias, consistencia lógica y rigor metodológico-crítico, solidez y excelencia, correspondencia con los fines y principios estatutarios, criterios de evaluación de los resultados y articulación con los sistemas de organización institucional. También Fallas, López y Meoño (1982) diferencian aspectos centrales sobre la naturaleza de su acción:

En la relación extensiva se parte de hombres concretos que realizan tareas concretas por medio de la inserción en un sector social determinado. Sin embargo, para que ello constituya una verdadera actividad académica, la relación extensiva no puede considerarse en forma aislada e independiente de la estructura académica. Más bien, por el carácter científico que prevalece en nuestras actividades, la relación extensiva ha de estar integrada en un universo de tareas académicas, articuladas en varios niveles fundamentales: el plan, el programa y el proyecto. (p. 135)

A su vez, los textos son exhaustivos en caracterizar el sentido de integralidad de la extensión como acción sustantiva, esto es su carácter de articulación natural con las otras formas, la investigación y la docencia. Por ejemplo, Fallas, López y Meoño (1982) consideran que:

Para que se dé una vinculación orgánica entre la extensión y la estructura de la Universidad, el programa de extensión debe formar parte de un plan global que contemple las relaciones de la extensión, la investigación y la docencia. Las relaciones en el sistema que caracterizan la relación extensiva se convierten en un elemento de un sistema más amplio que expresa la vinculación especial de las áreas académicas. (p. 139)

Desde la perspectiva señalada, estos autores le otorgan a la extensión un carácter dinamizador del quehacer sustantivo. Complementario a esta postura, Paniagua (2015) concluye también que:

...la extensión e investigación, en este caso particularmente, son procesos complementarios cíclicos. Por una parte, se inició con un proceso de investigación, el cual se adecuó con prácticas extensionistas tales como: identificación de actores claves en la comunidad, comunicación 
comunitaria, detección de problemas sociales, entre otros. Por otra parte, al inmiscuirse en estas experiencias y realidades, se identificaron nuevos enfoques investigativos. (p. 76)

Un argumento que se diferencia de los anteriores, especialmente por su carácter crítico respecto a la ubicación de la extensión en el marco del quehacer universitario, es el de Rivera (1983), quien señala que esta se ha supeditado a la investigación, y con ello pierde el carácter propio y la relega a un papel secundario:

La extensión es reducida a un momento o aspecto de la investigación científica, a saber: la verificación de los postulados teórico-metodológicos que ésta ha generado. De esta manera, la extensión ya no es una actividad académica específica, con sus propios objetivos, métodos y teorías y, fundamentalmente, con una forma particular y propia de vincular a la Universidad con la sociedad, sino que es parte de la investigación y se la confunde con un momento fundamental de ésta, como es el de la verificación o sometimiento de los productos de la investigación científica a la realidad para comprobar su adecuación o no a ésta. (p. 139)

Desde esta perspectiva, la extensión se expresa como el resultado de un proceso integrador del quehacer universitario y como una tarea imperativa de la comunicación universidad-sociedad.

\section{Vinculación social orgánica}

Esta última perspectiva expresa argumentos relacionados con los destinatarios metay las metodologías particulares para el ejercicio delaextensiónuniversitaria. Los principales exponentes de esta son Coto (2012), Paniagua (2015), Solano (2013), León F (2011), Parada (2015), Ramírez (2011), Arias (2015), Meza (2014) y Loría (2012). Los textos analizados comparten prácticamente dos criterios centrales: el énfasis del trabajo directo con las poblaciones de mayor vulnerabilidad y el carácter eminentemente participativo y democrático de esa vinculación. Proponen que es a través del diálogo verdadero, entendido como praxis social humanizadora (Freire, 1965), que la universidad logra impulsar cambios sociales sustantivos y que estos, prioritariamente, deben atender (problematizar) a las poblaciones que históricamente han quedado relegadas y marginadas del crecimiento y desarrollo.

Respecto a estas se registra una clara vocación por las ubicadas en las áreas rurales y los territorios costeros, enfatizando en los grupos sociales vulnerabilizados: mujeres, niños, indígenas, campesinos, pescadores, entre 
otros. La articulación con las distintas formas de la organización social aparece como el elemento articulador de la extensión universitaria, de ahí la invocación al trabajo con asociaciones de desarrollo comunal, instituciones públicas, centros educativos, organizaciones sindicales, cooperativas, tal como queda de manifiesto en la siguiente referencia, alusiva a un proyecto de extensión de la UNA desarrollado con un grupo de Municipalidades:

La gestión del proceso local se entiende como el saber hacer las cosas de la mejor manera posible por las instituciones y los gobiernos locales, con el máximo de beneficio para las comunidades. Es la capacidad de generar cambios, modificando las situaciones actuales, promoviendo la participación creativa e innovadora de los actores sociales, como gestores de su propio desarrollo, transformando el territorio. (Gamboa, 2013, p. 309)

De igual forma, según lo plantea Sobrado (2011):

Esta forma de desarrollo local basada en diversas formas de cooperación permite aprovechar los estímulos externos para propiciar un desarrollo endógeno impidiendo o mitigando la devastación y desequilibrios que generan las transnacionales cuando actúan el libre mercado significa retirar la escalera de la autonomía a los países emergentes. (p. 103)

La vinculación con pequeños productores y emprendedores expresa un acercamiento con las formas organizativas de tipo empresarial, dejando evidencia de una forma un tanto diferente de la extensión universitaria, más cercana al desarrollo de competencias vinculadas al fortalecimiento de las formas productivas dominantes socialmente. Por ejemplo, Parada (2015) expresa:

Las universidades aportan al bienestar, a través del vínculo directo o indirecto con la sociedad, acciones que se concretizan en programas y proyectos de acción social y que son parte de la extensión universitaria. La vinculación entre la universidad y la sociedad, históricamente, se ha hecho a través de la extensión, entendida esta como una acción sustantiva que mejora las condiciones de vida de las familias y transforma la dinámica de las empresas, las cuales originan producción, empleo e inversión. (p. 3)

Como se mencionó, la participación consciente es el eje central de la vinculación, por eso se condiciona a su carácter intencionado y de retroalimentación, tanto entre sus integrantes como en la universidad. Tal como lo expone Gamboa (2013), por participación se entiende: 
...una forma de intervención social que le permite a los individuos reconocerse como actores que, al compartir una situación determinada, tienen la oportunidad de identificarse a partir de intereses, expectativas y demandas comunes y que estén en capacidad de traducirlas en forma de actuación colectiva con una cierta autonomía frente a otros actores sociales y políticos. (p. 308)

Las formas de llevar a cabo la vinculación universidad-sociedad, en el sentido descrito y que le aporta a ese carácter orgánico, están íntimamente asociadas a los principios de la metodología de la investigación acción participativa (IAP), impulsada por el sociólogo colombiano Orlando Fals Borda a inicios de los setenta. Al respecto, Goldemberg (1988) asevera:

El Proyecto de Desarrollo Social Urbano está concebido en el marco de los principios que sustenta la metodología de investigación participativa. Esto significa que la orientación general de las acciones tiende a potenciar la capacidad autogestionaria de los sectores populares mediante el fortalecimiento y desarrollo de sus propios instrumentos organizativos. (p. 248).

De igual forma, Aguirre (2017) expone que:

...un enfoque de abordaje en investigación-participación (IAP). Consiste en la producción de conocimiento sobre la problemática socio-ambiental, que permite la sistematización de experiencias con el propósito de cambiar la situación actual y redireccionar el rumbo según las necesidades encontradas; lo que se efectúa mediante un proceso participativo que involucra al equipo de trabajo y los actores locales identificados. (p. 206)

Destacan en esta perspectiva una importante variedad de estrategias y metodologías plasmadas en los documentos analizados, entre las que, a manera de ejemplo, se pueden citar la metodología de capacitación masiva y laboratorio organizacional (Sobrado, 2012), los métodos rápidos de análisis (Coto, 2016), la planificación del desarrollo sostenible de una microrregión (PDSM) (Aguirre, 2016) y el «Proyecto de desarrollo social urbano» (Goldemberg, 1988). Resalta también la máxima, tal como se expone en el documento producido por la Unidad Coordinadora de Extensión de la Facultad de Ciencias Sociales (1986): 
La metodología de extensión debe comprender una adecuada interrelación entre la práctica y la teoría, de tal manera que se produzca la creación de nuevos conocimientos. La tendencia al practicismo, presente en algunas oportunidades, impide sistematizar las experiencias y ordenar los datos obtenidos en el contacto con la realidad. (p. 201)

Finalmente, se logró identificar dentro del mapa-dibujo, en asociación con las diferentes perspectivas discutidas, al grupo de autores cuya producción fue analizada (ver figura 2) con el propósito de mostrar una visión, también provisoria, de estos en el contexto general del discurso académico sobre la extensión.

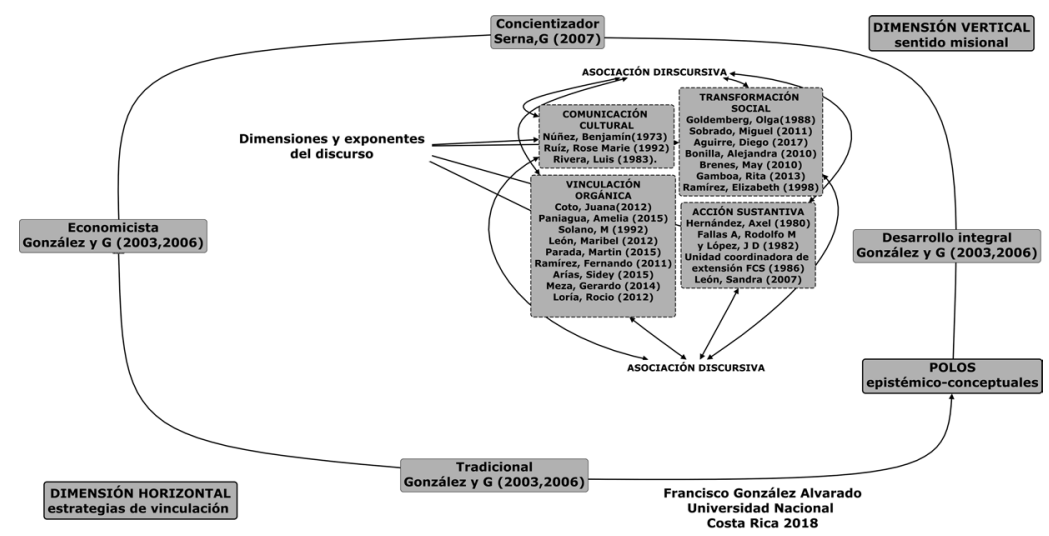

Figura 2. Mapa-dibujo de los autores responsables de la producción académica analizada dentro del territorio discursivo.

Fuente: Francisco González Alvarado (2018).

\section{Conclusiones}

La cartografía del discurso académico sobre la extensión en la UNA permitió identificar cuatro perspectivas o visiones de este particular quehacer universitario. Es importante señalar que, a pesar de que estas recogen visiones de carácter más complementario que disonante, su identificación y análisis, así como su ordenamiento en el marco del territorio discursivo construido, facilitó reconocer aspectos centrales, aunque provisorios, de lo que puede denominarse naturaleza y carácter propio de la extensión universitaria en la Universidad Nacional. Esa representación de alguna manera circunstancial y temporal está estrechamente ligada a la evolución histórica del concepto de extensión que, siguiendo a Stein (2018), «ha estado sujeto a diversos enfoques que, sin lugar a dudas, guardan una 
URL: http://www.revistas.una.ac.cr/index.php/dialogo/index

CORREO ELECTRÓNICO: universidadendialogo@una.cr

DOI: http://doi.org/10.15359/udre.9-2.4

relación directa con los diferentes modelos o corrientes ideológicas construidos en torno a la universidad, en diferentes contextos históricos y geográficos» (p. 23).

Una primera perspectiva identificada es la denominada transformación social, que expresa su vinculación con los principios fundacionales de la universidad y también explicita el propósito central, el reconocimiento y posterior desarrollo de estrategias de inserción universitaria orientadas al cambiomodificación de las condiciones sociales, económicas, políticas y culturales de las comunidades más vulnerables del país. La comunicación cultural expone el papel de la extensión como una forma de comunicación-inserción social y distingue a la vez diferentes niveles en la consecución de esa tarea misional, que es considerada como una opción ético-político; de igual manera, marca los límites respecto a otras formas típicamente orientadas a la mera difusión social del quehacer universitario. En este punto, diferencia las formas de extensión típicamente instrumentalistas de aquellas verdaderamente transformadoras.

Otra de las perspectivas identificadas, la denominada acción sustantiva, define la extensión universitaria como una de las acciones centrales del quehacer particular de la universidad, establece las condiciones capitales para que sea desarrollada y puntualiza en su carácter interdisciplinario. De igual manera, rechaza las categorizaciones que la ubican en un plano residual respecto a las otras formas de la acción sustantiva, es decir, la investigación y la docencia y aboga por una postura de interrelación dinámica, permanente e intencional respecto a estas.

Finalmente, la vinculación social orgánica deja evidencia señera respecto a los sectores sociales considerados de atención prioritaria por parte del ejercicio de la extensión en la UNA; estos son los grupos más excluidos del modelo de desarrollo social costarricense. A su vez, expone las metodologías y sus condiciones para lograr una verdadera comunicación-inserción social, fuertemente vinculadas a la investigación acción participativa y sus diversos enfoques.

Es importante destacar que las perspectivas gravitan en el mapa con mayor acercamiento hacia los polos epistémico-conceptuales vinculados con los modelos de la extensión universitaria concientizador e integral, y en menor medida hacia el tradicional, mostrando un fuerte alejamiento del economicista, a pesar de que se constatan diferencias argumentativas al interior de las dimensiones que revelan concomitancias puntuales con este último modelo. Esta determinación identifica una parte central de la naturaleza y carácter propio de la extensión en la UNA, el sentido esencialmente político y social de su praxis y está absolutamente influenciada por los postulados filosóficos, ontológicos, éticos y epistémicos fundacionales de esta casa de estudios superiores. 


\section{Referencias bibliográficas}

Aguirre, D. (junio-diciembre, 2016). La extensión de la UNA y su impacto en el desarrollo de las comunidades rurales: aportes al desarrollo autogestionario de la comunidad de Barra de Colorado, Pococí, Limón. Universidad en Diálogo, 6(2), 205-240. https://doi.org/10.15359/ udre.6-2.12

Aguirre, D. Arboleda, E. Hernández, G. Méndez, A. Montero, S. Montero, F. Sánchez, H. (Julio-Septiembre, 2017). Unidades productivas integradas asociadas con los saberes ancestrales indígenas cabécar: desde la perspectiva de extensión universitaria. Revista Granmense de Desarrollo Local, 1(2), 1-7. https://revistas.udg.co.cu/index.php/redel/ article/view/460/759

Arias, S. Valverde, K. Rodríguez. Ramírez, S. (Julio-Diciembre, 2015). Producción de toplancton para la investigación y la docencia en larvicultura de camarones, moluscos y peces, 2008-2013. Universidad en Diálogo, 5(2), 85-99

Bonilla, A. (2010). Más desposeídas que propietarias. San José, Costa Rica: Fondo de Naciones Unidas para el Desarrollo.

Brenes, May. (2006). Proyecto Desarrollo Rural Integral de las Mujeres en el Golfo de Nicoya Forjando un sueño y recopilando historias. Heredia, Costa Rica: Instituto de Estudios de la Mujer. Universidad Nacional.

Pérez, C., Coto J., Salgado V., Herrera J., Fernández José., Benavides C. (Enero-Junio, 2013). Tratamiento de aguas residuales con tecnologías alternativas en una pequeña unidad doméstica-productiva. Uniciencia, 27(1), 319-331.

Diez Tetamanti, J. M. y Chanampa, M. E. (2016). Perspectivas de la Cartografía Social, experiencias entre extensión, investigación e intervención social. +E: Revista de Extensión Universitaria, 6, 84-94. Santa Fe, Argentina: Ediciones UNL. https://doi.org/10.14409/extension.v1i6.6316

Fallas, A., López, J. y Meoño, R. (1982). La extensión como actividad académica. Departamento de Filosofía, Universidad Nacional.

Freire, P. (1965). La educación como práctica de la libertad. España: Siglo XXI. 
Gamboa, R. (2013). Abordaje de problemáticas sociales latinoamericanas desde la academia: competencias del extensionista universitario. Congreso: El Viejo Mundo y el Nuevo Mundo en la Era del Diálogo. Ankara, Turquía.

González, G. R. y González, M. (2003). Extensión universitaria: principales tendencias en su evolución y desarrollo. Revista Cubana de Educación Superior, 23(1), 15-26.

González, G. R. y González, M. (2006). Extensión universitaria: una aproximación conceptual desde la perspectiva cubana. Revista Cubana de Educación Superior, 26(2): 69-76.

Goldemberg, O. (1988). Apuntes hacia una definición metodológica del proyecto social urbano. Revista Abra, 8(9-10), 241-249.

Hernández, A. (1980). El carácter académico de la extensión en la Universidad Nacional y sus principales implicaciones. Ponencia. Seminario acerca de la investigación y la extensión en la Facultad de Ciencias Exactas y Naturales, de la Universidad Nacional. Heredia.

León, F M. (Julio-Diciembre 2011). Mujer adulta mayor, derechos, políticas y lineamientos y el Programa de Atención Integral de la Persona Adulta Mayor (PAIPAM). Revista Casa de la Mujer, 20(2), 119-131. http://45.162.204.69/index.php/mujer/article/view/6508/6567

León, S. (2007). Seminario Latinoamericano de Educación Rural. Revista Electrónica Educare, 2(Extraordinario), 13-15. https://www.revistas. una.ac.cr/index.php/EDUCARE/article/view/1369

Loría, R. (2012). Los límites socioculturales al espacio de recolectores inmigrantes de café. Revista Reflexiones, 91(1), 225-263.

Meza, G. E. (Enero-Diciembre 2014). Acercamiento a la poética musical del compositor guatemalteco Jorge Sarmientos. Ístmica, 17, 65-81. https:// www.revistas.una.ac.cr/index.php/istmica/article/view/7072

Núñez, B. (1974). Universidad necesaria. Heredia: Departamento de Publicaciones, Universidad Nacional.

Ortiz, M. y Morales, M. (2011). La extensión universitaria en América Latina: concepciones y tendencias. Universidad Militar Nueva Granada, Colombia. 
Paniagua, A. (enero-junio, 2015). Claves metodológicas de la extensión universitaria. Universidad en Diálogo, 5(1). 73-86.

Parada, M. (2015). Metodología de extensión para crear emprendimientos productivos: caso de Isla Venado, Golfo de Nicoya, Costa Rica. Economía y Sociedad, 20(48), 1-22. https://doi.org/10.15359/eys.20-48.2

Ramírez, E. (1998). Laguna Hule: experiencia de un modelo ecoturístico comunitario. Revista Geográfica de América Central, 2(35), 9-22.

Ramírez, F, Calderón R., Sánchez. L. (Julio-Diciembre, 2011). El sistema productivo apícola: una alternativa para el desarrollo sostenible de la Región Central Sur de Costa Rica. Universidad en Diálogo, 1(2), 31-54

Rivera,L.(1983).Práctica extensiva en elDepartamento de Filosofia: concepciones y realizaciones. Departamento de Filosofía, Universidad Nacional.

Ruiz, R. M. (1992). Hacia una difusión cultural universitaria. Heredia, Costa Rica: Departamento de Publicaciones, Universidad Nacional.

Ruiz, R. M. (enero-junio, 2011). Extensión: transformaciones vitales en la relación universidad-comunidad. Universidad en Diálogo, 1(1), 11-28

Santos, J. (2010). Conflicto de representaciones. América Latina como lugar para la filosofia. Santiago, Chile: Fondo de Cultura Económica.

Serna, G. (2007). Misión social y modelos de extensión universitaria: del entusiasmo al desdén. Revista Iberoamericana de Educación, 43, 3-25.

Sobrado, M. (enero-junio, 2011). Desarrollo local y ajuste organizacional. Universidad en Diálogo, 1(1), 74-103

Sobrado, M. (Enero-Junio de 2012). La metodología de capacitación masiva y el desarrollo local y regional. Universidad en diálogo, 2(1), 43-58. https://www.revistas.una.ac.cr/index.php/dialogo/article/view/5627

Alfaro C., Pérez P., Solano M. (Junio, 2013). Saneamiento de aguas residuales mediante humedales artificiales en el Museo de Cultura Popular de la Universidad Nacional. Revista de Ciencias Ambientales, 45(1), 63-71. https://doi.org/10.15359/rca.45-1.6 
Stein, N. S. (2018). La extensión-comunicación universitaria disputando el lugar de la investigación como fuente de conocimiento. Un recorrido histórico hasta los enfoques actuales. Revista de Extensión Universitaria, 8(9), 14-37. https://doi.org/10.14409/extension.v8i9.Jul-Dic.7866

Tello, C. y Gorostiaga, J. M. (julio-diciembre, 2009). El enfoque de la cartografía social para el análisis de debates sobre políticas educativas. Práxis Educativa, 4(2), 159-168. https://doi.org/10.5212/ PraxEduc.v.4i2.159168

Tello, C. y Gorostiaga, J. M. (maio-ago, 2011). Globalización y reforma educativa en América Latina: un análisis inter-textual. Revista Brasileira de Educação, 16(47), 363-388. https://doi.org/10.1590/ S1413-24782011000200006

Unidad Coordinadora de Extensión (1986). Resoluciones del Seminario sobre Experiencias Metodológicas en los Proyectos de Extensión de la Facultad de Ciencias Sociales de la Universidad Nacional. Facultad de Ciencias Sociales, Universidad Nacional.

Vicerrectoría de Extensión (2016). Reconocimiento al extensionista destacado. Universidad Nacional. 
